\title{
Distinct progenitor populations in skeletal muscle are bone marrow derived and exhibit different cell fates during vascular regeneration
}

\author{
Susan M. Majka, ${ }^{1,2,3}$ Kathyjo A. Jackson,, ${ }^{1,3}$ Kirsten A. Kienstra, ${ }^{1,3}$ Mark W. Majesky,,5 \\ Margaret A. Goodell, ${ }^{1,3,6,7}$ and Karen K. Hirschi1 ${ }^{1,2,3,4}$ \\ ${ }^{1}$ Department of Pediatrics, \\ ${ }^{2}$ Children's Nutrition Research Center, \\ ${ }^{3}$ Center for Cell and Gene Therapy, \\ ${ }^{4}$ Department of Molecular and Cellular Biology, \\ ${ }^{5}$ Department of Pathology, \\ ${ }^{6}$ Department of Immunology, and \\ ${ }^{7}$ Department of Molecular and Human Genetics, Baylor College of Medicine, Houston, Texas, USA
}

\begin{abstract}
Vascular progenitors were previously isolated from blood and bone marrow; herein, we define the presence, phenotype, potential, and origin of vascular progenitors resident within adult skeletal muscle. Two distinct populations of cells were simultaneously isolated from hindlimb muscle: the side population (SP) of highly purified hematopoietic stem cells and non-SP cells, which do not reconstitute blood. Muscle SP cells were found to be derived from, and replenished by, bone marrow SP cells; however, within the muscle environment, they were phenotypically distinct from marrow SP cells. Non-SP cells were also derived from marrow stem cells and contained progenitors with a mesenchymal phenotype. Muscle SP and non-SP cells were isolated from Rosa26 mice and directly injected into injured muscle of genetically matched recipients. SP cells engrafted into endothelium during vascular regeneration, and non-SP cells engrafted into smooth muscle. Thus, distinct populations of vascular progenitors are resident within skeletal muscle, are derived from bone marrow, and exhibit different cell fates during injury-induced vascular regeneration.
\end{abstract}

J. Clin. Invest. 111:71-79 (2003). doi:10.1172/JCI200316157.

\section{Introduction}

Postnatal neovascularization, in response to tissue injury and remodeling, was thought previously to occur solely via the proliferation and migration of endothelial cells from preexisting vasculature. However, recent studies suggest that vascular progenitors resident within peripheral blood $(1,2)$ and bone marrow (3-6) significantly contribute to injury-induced (4-6) and pathology-induced (7) neovascularization. We aimed to determine whether vascular progenitors reside within adult tissues other than bone marrow and

Received for publication on June 11, 2002, and accepted in revised form on October 8, 2002.

Address correspondence to: Karen K. Hirschi, Center for Cell and Gene Therapy, Baylor College of Medicine, Houston 77030, Texas, USA. Phone: (713)-798-7771; Fax: (713) 798-1230;

E-mail: khirschi@bcm.tmc.edu.

Conflict of interest: The authors have declared that no conflict of interest exists.

Nonstandard abbreviations used: side population (SP); plateletendothelial cell adhesion molecule-1 (PE-CAM-1); transcription factor Scl/Tal-1 (Tal 1); VEGF receptor-1 (Flt-1); VEGF receptor-2 (Flk-1); angiopoietin-1 (Ang-1); tyrosine kinase with immunoglobulin and epidermal growth factor homology-1 (Tie-1); vascular-endothelial cadherin (VE-cadherin); smooth muscle $\alpha$ actin (SM- $\alpha$-actin); fluorescein-di- $\beta$-Dgalactopyranoside (FDG); tibialis anterior (TA). to what extent they contribute to tissue-specific, injuryinduced vascular regeneration.

We focused on defining the vascular potential of stem cells resident within adult skeletal muscle that would be easily accessible for autologous cell therapies and tissue-engineering applications (8). Muscle-derived cells have been shown to exhibit diverse stem cell activities, including the regeneration of injured muscle fibers (9), as well as the reconstitution of hematopoietic cell lineages upon bone marrow transplantation $(10,11)$. In recent studies, we Percoll-fractionated the skeletal muscle-derived cells, subjected the dissociated cells to Hoechst 33342 dye staining and FACS, and isolated a population of cells known as "side population" or SP cells, which actively efflux the Hoechst dye. By bone marrow transplantation of marked muscle-derived SP cells into lethally irradiated recipients, we determined that the hematopoietic potential of skeletal muscle resides within the SP population (S. McKinney-Freeman et al., manuscript submitted for publication). We have demonstrated previously that SP cells derived from bone marrow contribute to the regeneration of vascular endothelium during injury-induced neovascularization (4), in addition to reconstituting blood (12); thus, we hypothesized that the muscle-derived SP cells would have similar vascular potential. 
We also aimed to determine whether other "non-SP" cells within the muscle could serve as vascular progenitors. Non-SP cells do not efflux the Hoechst dye as efficiently as SP cells and, upon FACS analysis, appear as a distinct population of cells adjacent to the SP population. Muscle-derived non-SP cells do not have the potential to reconstitute blood in lethally irradiated bone marrow transplant recipients (S. McKinney-Freeman et al., manuscript submitted for publication).

In the studies presented here, we defined the phenotype of both SP and non-SP cells within muscle tissue, relative to the known characteristics of embryonic vascular precursors, as well as the phenotype of bone marrow SP cells, which we found gave rise to both of these muscle populations. We then examined their potential to regenerate vascular endothelium and smooth muscle via direct injection into injured muscle tissue. We found that these two phenotypically distinct populations of stem cells resident within skeletal muscle differentially contributed to vascular regeneration in response to injury: muscle-derived SP cells gave rise to vascular endothelium, and non-SP cells regenerated vascular smooth muscle during injury-induced neovascularization of skeletal muscle.

\section{Methods}

Isolation of skeletal muscle progenitors. Skeletal muscle cells were isolated from 6- to 8-week-old C57Bl/6, C57Bl/6, Rosa26, or FVB/N-TgN(Tie-2LacZ) mice (The Jackson Laboratories, Bar Harbor, Maine, USA). Hindlimb skeletal muscles were excised, minced, and digested for 30 minutes at $37^{\circ} \mathrm{C}$ with type II collagenase $(0.2 \%$; Worthington Biochemical Corp., Lakewood, New Jersey, USA). The digested tissue was pelleted and resuspended, triturated in HBSS, and passed through a $40-\mu \mathrm{m}$ filter to yield a single-cell suspension. The cells were then size-fractionated by layering onto a $40 / 70 \%$ Percoll gradient and centrifuging for 20 minutes at $1,000 \mathrm{~g}$ at room temperature. Cells at the interface were subjected to Hoechst staining and FACS to isolate skeletal muscle SP and non-SP cells. Extensive details of SP cell isolation can be found in a previous publication (12) or downloaded (www.bcm.tmc.edu/genetherapy/goodell).

Briefly, Percoll-fractionated muscle cells were suspended at $10^{6}$ nucleated cells per milliliter in DMEM with $2 \%$ FCS $/ 10 \mathrm{mM}$ HEPES buffer $/ 5 \mu \mathrm{g} / \mathrm{ml}$ Hoechst 33342 and incubated for 90 minutes at $37^{\circ} \mathrm{C}$. Cells were then incubated with propidium iodide $(2 \mu \mathrm{g} / \mathrm{ml})$ to label nonviable cells and subjected to FACS. The fluorescent profile of Hoechst-stained cells is shown in Figure 1; propidium iodide-positive (dead) cells were excluded. Muscle SP cells actively efflux the Hoechst dye and appear as a distinct population of cells on the side of the profile; hence, the name SP cells. Non-SP cells constitute a distinct and reproducible population of cells that typically contains approximately $70-80 \%$ of the viable Percoll-fractionated muscle cells (see Figure 1). Cell analysis and collection was performed on a triple laser MoFlow instrument (Cytomation Inc., Fort

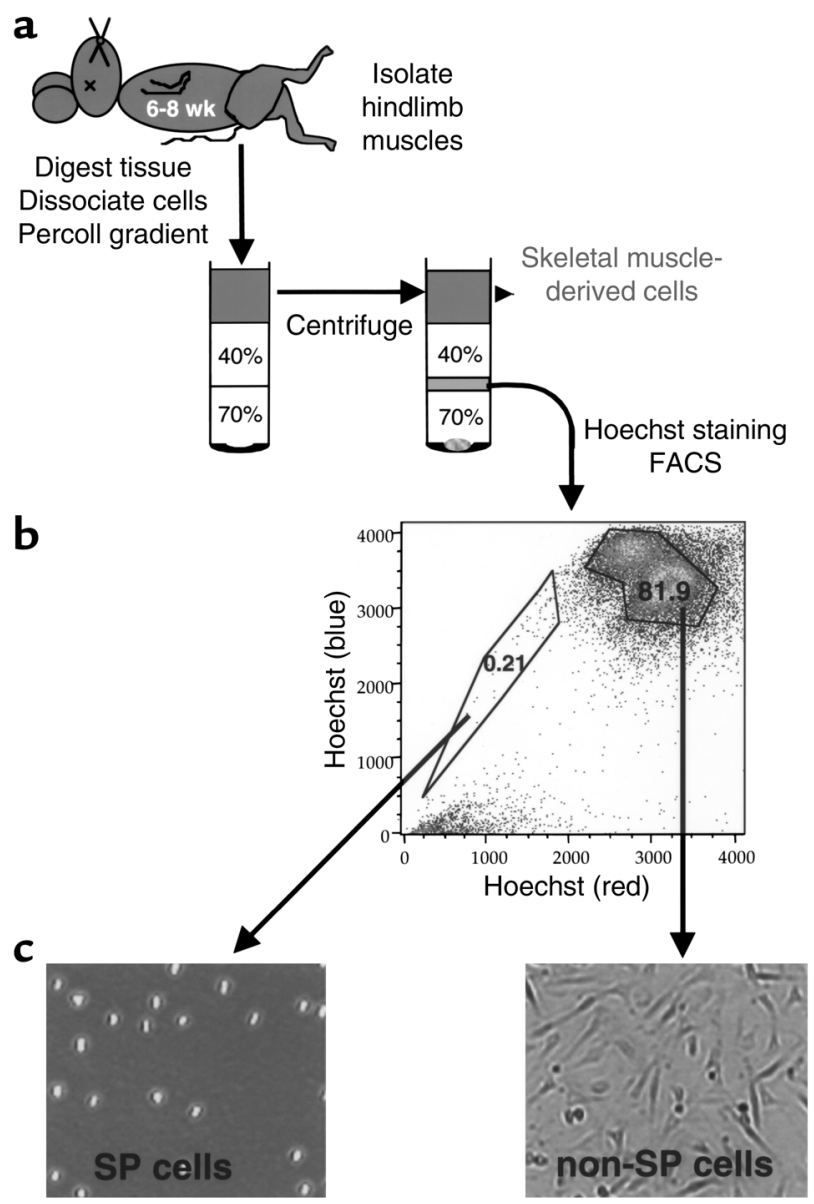

Figure 1

Isolation of murine skeletal muscle progenitors. (a) Skeletal muscle stem cells were isolated from 6 - to 8 -week-old C57BI/6 or Rosa26 mice, fractionated on a Percoll gradient, stained with Hoechst 33342 dye, and subjected to FACS. SP and non-SP cells were clearly discernable based on Hoechst dye exclusion, and the distinct populations were isolated via FACS. (b) In this representative sample, the SP population was $0.21 \%$, and the non-SP was $81.9 \%$ of the total viable cells. (c) Both SP and non-SP populations were cultured overnight, and their distinct morphologies were revealed upon microscopic examination of the cells in culture. The SP cells were found to be of uniform morphology and nonadherent. The non-SP population contained predominantly adherent, spindle-shaped cells.

Collins, Colorado, USA) and data analyzed using FlowJo Software (Treestar Software, San Carlos, California, USA). Cytospin preparations were also made from isolated muscle SP and non-SP cells.

Analysis of stem cell populations for vascular marker expression. Total RNA was extracted from Percoll-fractionated skeletal muscle-derived cells, muscle SP and non-SP cells, and bone marrow SP cells using Trizol (Invitrogen Life Technologies Inc., Carlsbad, California, USA). Reverse transcription was performed on $50 \mathrm{ng}$ of DNAse-treated RNA, using the suggested protocol for the Superscript II kit (Invitrogen Life Technologies Inc.). Two-step PCR was performed using primers for the following genes, as reported previously (4): CD34; plateletendothelial cell adhesion molecule-1 (PE-CAM-1); 
transcription factor Scl/Tal-1 (Tal 1); VEGF receptor-1 (Flt-1); VEGF receptor-2 (Flk-1); VEGF-A; angiopoietin-1 (Ang-1); tyrosine kinase with immunoglobulin and epidermal growth factor homology-1 (Tie-1); vascularendothelial cadherin (VE-cadherin); smooth muscle alpha actin (SM- $\alpha$-actin); calponin; desmin; and GAPDH.

Primers against other genes included: hepatocyte growth factor/scatter factor receptor (c-met), $5^{\prime}$-GAATGTCGTCCTACACGGCC-3' forward, 5'-CACTACACAGTCAGGACACTGC-3' reverse; and ICAM-2, 5'-CATATGGTCCGAGAAGCAGA-3' forward, 5'-TGCACTCAATGGTGAAGTCT- $3^{\prime}$ reverse. Analysis using each primer set was performed on three to five independent RNA isolates from each cell population. Positive (RNA isolated from whole bone marrow or muscle tissue), negative (no reverse transcriptase enzyme added to RT reaction), and loading (amplification of GAPDH) controls were included in all runs. Furthermore, the amplicon generated from each primer set, using control muscle- or bone marrow-derived RNA, was cloned into the PGEM-T vector, using the pGEM-T EasyVector System I (Promega Corp., Madison, Wisconsin, USA) and sequenced to verify that the primers amplify the intended gene.

Cells were also examined via flow cytometry for the expression of cell surface proteins. The muscle SP and non-SP cells were isolated as described above, then pelleted by centrifugation and resuspended at $10^{8} \mathrm{cells} / \mathrm{ml}$ in cold HBSS containing 2\% FCS and 10 mM HEPES buffer $\left(\mathrm{HBSS}^{+}\right)$. Cells were incubated with primary Ab's for 20 minutes, washed in excess $\mathrm{HBSS}^{+}$, and then incubated with secondary reagents, as needed, for $20 \mathrm{~min}$ utes before a final wash and resuspension in $\mathrm{HBSS}^{+}$. Primary Ab's (all from PharMingen, San Diego, California, USA) included: CD45 (clone 30-F11), CD34 (clone 49E8), Sca-1 (E13-161.7), Flk-1 (clone Avas 12 $\alpha 1$ ), ICAM-2 (clone 3C4), PE-CAM-1 (clone MEC 13.3), and VE-cadherin (clone 11D4.1), either conjugated to PE or FITC, or followed by staining with PE- or FITC-conjugated secondary Ab's (Vector Laboratories, Burlingame, California; PharMingen). Expression of Tie-2 protein was examined by fluorescein-di- $\beta$-D-galactopyranoside (FDG) (FluoReporter LacZ Flow Cytometry Kit; Molecular Probes Inc., Eugene, Oregon USA) loading, via hypotonic shock, of the skeletal muscle SP and non-SP cells isolated from FVB/N-TgN(Tie-2LacZ) that express $L a c Z$ under the control of the Tie-2 promoter(13). FDGloaded cells were subjected to FACS to determine $\beta$-gal activity levels, as evidenced by the generation of a fluorescent cleavage product, which directly reflects $L a c Z$ expression driven by Tie- 2 transcriptional activation in the SP and non-SP cells.

Species-specific secondary Ab's (anti-rat PE or FITC) and isotype-matched Ab's (mouse anti-rat IgG2b\%, PharMingen clone G15-337; and mouse anti-rat IgG2aא, PharMingen clone R35-95) were used as negative controls for flow cytometry gating and analyses. All analyses were performed on four independent cell isolations using a triple laser MoFlow instrument (Cytomation Inc.), as described (4). Data generated were analyzed using FlowJo Software (Treestar Software), and are presented as the mean \pm SD of the four independent experiments.

Determination of the origin of vascular progenitors within skeletal muscle. We aimed to determine whether the SP and non-SP cells resident within skeletal muscle were derived from bone marrow stem cells. To do so, LacZmarked bone marrow-derived SP cells were isolated from Rosa 26 mice, as described above for muscle SP cell isolation and elsewhere (12), and transplanted via retro-orbital injection into lethally irradiated genetically matched $\mathrm{C} 57 \mathrm{Bl} / 6$ recipients, as described previously (4). Engraftment of LacZ-marked SP cells into the bone marrow of recipients was monitored via analysis of peripheral blood for FDG activity using FACS, as described above. At 5 or 12 months after transplantation, the hindlimb skeletal muscles of stably engrafted mice (three mice for each time point) were excised, digested, stained with Hoechst dye, loaded with FDG substrate via hypotonic shock. The cells were then subjected to FACS to isolate SP and non-SP populations and to detect $\beta$-gal activity in each population, as described above, to determine what proportion of each was expressing the $L a c Z$ gene and, hence, derived from bone marrow.

Skeletal muscle injury and stem cell delivery. To test the vascular potential of muscle-derived SP and non-SP cells in vivo, we independently injected each LacZ-marked population into injured muscle tissue and, after 4 weeks, analyzed the repaired tissue for engraftment of marked SP and non-SP cells into the vasculature. Recipient mice for the injury models were prepared as follows. Briefly, 6- to 8-week-old genetically matched C57Bl/ 6 mice, either 8 or 24 hours prior to injection of stem cells, were anesthetized with Avertin, and $25 \mu \mathrm{l} \mathrm{of} \mathrm{a} 1 \mathrm{mg} / \mathrm{ml}$ cardiotoxin Naja mossambica mossambica (Sigma-Aldrich, St. Louis, Missouri, USA) was injected lengthwise into both tibialis anterior (TA) muscles. This mixture of snake toxins induces the degeneration of muscle fibers (14); muscle injury was associated with hindlimb inflammation and limping for up to 48 hours after injury. The right limb TA muscle was then injected with either 500,000 non-SP or 2,000 SP cells, and the left leg was injected with Hanks solution alone. After 4 weeks, the lower hindlimb musculature was harvested and processed for frozen sectioning and immunohistochemical analysis. Four animals were analyzed for each SP and non-SP cell engraftment study; control and experimental muscle tissue from each of the animals was evaluated.

Immunohistochemical analysis of experimental tissues. Frozen sections $(5 \mu \mathrm{m})$ of experimental skeletal muscle tissue were immunostained with Ab's against desmin (DAKO A/S, Glostrup, Denmark; 1:500) and ICAM-2 (PharMingen, clone 3C4; 1:50) to identify smooth muscle and endothelial cells, respectively, within vascular structures. For desmin immunostaining, tissue was fixed for 30 minutes with $4 \%$ paraformaldehyde prior to primary $\mathrm{Ab}$ incubation. For ICAM-2 immunostaining, tissue was similarly 


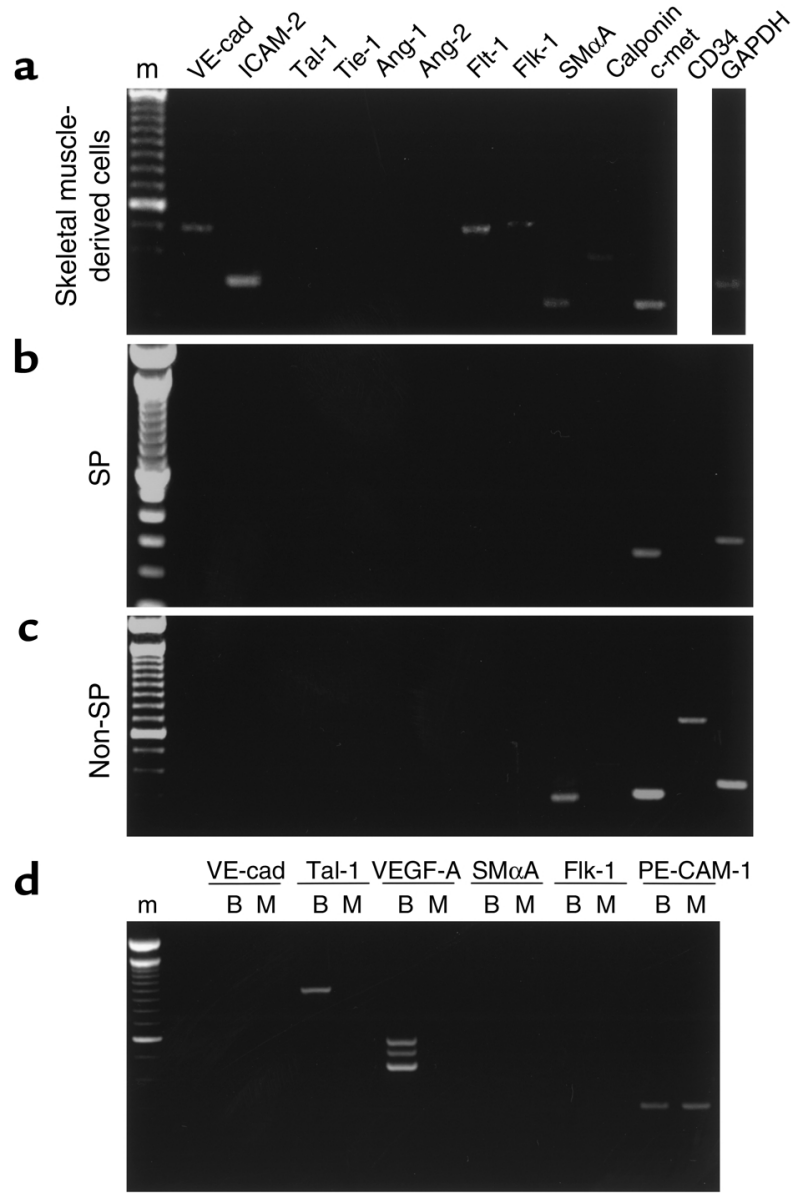

Figure 2

Analysis of vascular-specific gene expression in skeletal muscle-derived cells. RT-PCR analysis was performed on total RNA (50 ng/sample) isolated from distinct populations of skeletal muscle cells: (a) Percollfractionated skeletal muscle cells; (b) muscle SP population; and (c) muscle non-SP population. (d) Bone marrow-derived SP cells were directly compared to skeletal muscle-derived SP cells. GAPDH was used as a loading control. Analyses using each primer set were performed on three to five independent RNA isolates from each cell population; representative data are shown. The amplicon generated from each primer set was cloned and sequenced to verify that the primers amplify the intended gene. VE-cad, VE-cadherin; SM $\alpha A$, SM- $\alpha$-actin; $\mathrm{m}$, marker; $\mathrm{B}$, bone marrow SP; $\mathrm{M}$, muscle SP.

fixed following the primary $\mathrm{Ab}$ incubation. Antimouse or anti-rat Alexa 488 or 594 secondary Ab's (Molecular Probes Inc.) were used for desmin and ICAM-2 immunostaining, respectively. Primary Ab's against $\beta$-gal (Cortex Biochem, San Leandro, California, USA; 1:500) and anti-rabbit Alexa 488 or $594 \mathrm{sec}-$ ondary Ab's were used to colocalize LacZ expression in vascular cells. Coverslips were mounted using Vectashield containing DAPI nuclear dye (Vector Laboratories), and slides were viewed using a Zeiss epifluorescent microscope equipped with differential interference contrast optics. As controls, skeletal muscle sections from C57Bl/6 and FVB/N-TgN(Tie-2LacZ) were similarly immunostained. Cytospins of musclederived SP and non-SP cells were also fixed with $4 \%$ paraformaldehyde and immunostained, as detailed above, using primary Ab's against desmin (DAKO A/S; 1:500), calponin (Sigma-Aldrich; 1:5,000), SM- $\alpha$ actin (DAKO A/S; 1:500), Flt-1 (Santa Cruz Biotechnology Inc., Santa Cruz, California, USA; 1:200), and PDGFR- $\beta$ (PharMingen; 1:250).

\section{Results}

Skeletal muscle contains two populations of vascular progenitors. Skeletal muscle cells were isolated (Figure 1a), stained with Hoechst 33342 dye, and subjected to FACS to yield a characteristic SP population, ranging from $0.1 \%$ to $0.5 \%$, and a non-SP population, ranging from $70 \%$ to $85 \%$ of viable cells (Figure $1 \mathrm{~b}$ ). The muscle-derived SP and non-SP populations were phenotypically distinct (Figure 1c): the SP cells were consistently round, nonadherent, and of uniform size; whereas, the non-SP population contained predominantly adherent, spindle-shaped cells.

The expression of genes reflective of progressive stages of vascular cell lineage commitment and differentiation was analyzed in the Percoll-fractionated muscle-derived cells, as well as muscle SP and non-SP fractions, using RT-PCR analysis. The Percoll-fractionated muscle cells expressed markers of both endothelial (VE-cad, ICAM-2, Flt-1, Flk-1) and smooth muscle lineages (SM- $\alpha$-actin, calponin) (Figure 2a). Highly purified skeletal muscle SP cells, in contrast, lacked markers of endothelial, smooth muscle, or mesenchymal cells and were CD34 negative (Figure $2 \mathrm{~b}$ ). The muscle non-SP cells expressed SM- $\alpha$-actin mRNA, characteristic of smooth muscle progenitors, or mesenchymal cells (15), as well as detectable levels of CD34 and sometimes calponin, but did not express desmin mRNA. Interestingly, both muscle-derived populations expressed the hepatocyte growth factor/scatter factor receptor gene, c-met (Figure 2, a-c), which is also expressed by the skeletal muscle progenitor cells, satellite cells, contained within skeletal muscle (16).

When marrow-derived and muscle-derived SP cells were analyzed in parallel, distinct differences in molecular expression were evident (Figure 2d). Bone marrow SP cells expressed genes reflective of embryonic hematopoietic progenitors (17) and vascular progenitors $(17,18,19)$, including PE-CAM-1 and Tal-1, as well as VEGF-A. Muscle SP cells, however, lacked expression of Tal-1 and VEGF-A (Figure 2d). As expected, both populations of SP cells lacked expression of genes reflective of endothelial (Flk-1, Flt-1, VE-cad, Tie-1) or smooth muscle (SM- $\alpha$-actin, calponin, Ang-2) lineages.

FACS analysis was performed in parallel with RT-PCR to examine the expression of the following cell surface proteins: CD45 (Figure 3a), Tie-2 (Figure 3b), Sca-1 (Figure 3c), and PE-CAM-1 (Figure 3d), as well as CD34, Flk-1, and VE-cadherin (not shown). Anti-CD45 labeled a high proportion of both SP $(63.6 \% \pm 17.7 \%)$ and non-SP $(92.9 \% \pm 2.8 \%)$ cells. Sca- 1 was also expressed in both populations (SP, $44.7 \% \pm 13.7 \%$; and non-SP, $26.8 \% \pm 18.5 \%$ ), although expression in the non-SP population was high- 

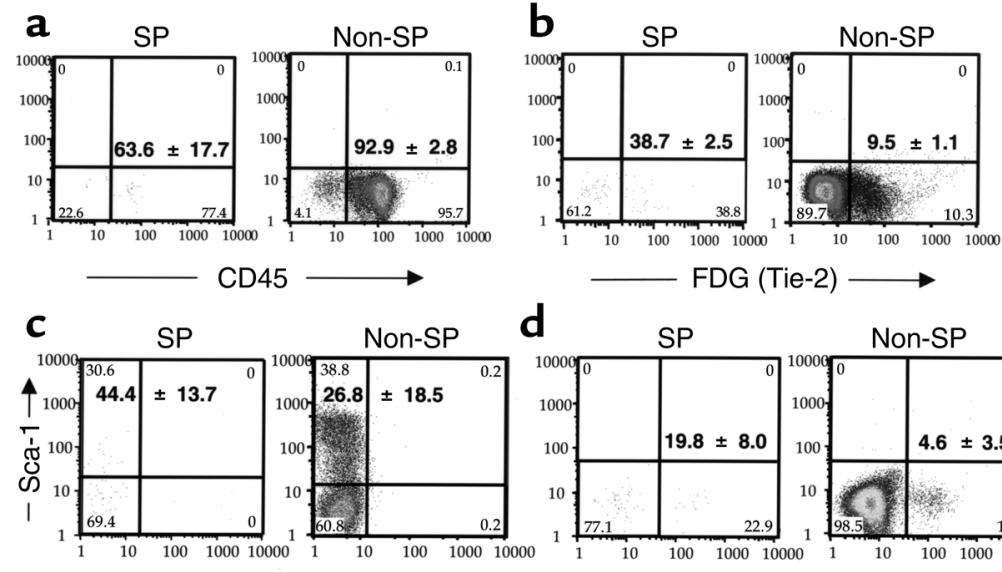
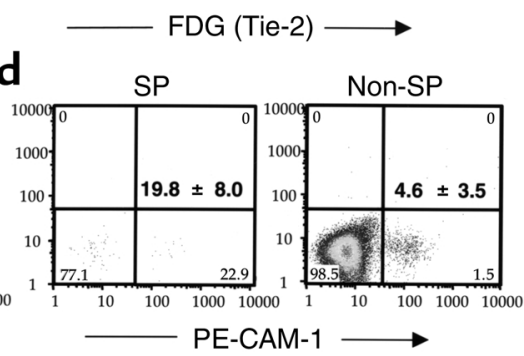

Figure 3

FACS analysis for vascular lineage markers in muscle SP and non-SP populations. FACS data were compiled from four experiments, and the expression of specific proteins, displayed as mean \pm SD from these independent experiments, are shown: (a) CD45; (b) Tie-2, as evidenced by FDG cleavage, indicating $\beta$-gal activity driven from the Tie- 2 promoter; (c) Sca-1; and (d) PE-CAM-1. ly variable. The SP population exhibited a higher proportion of Tie-2-positive cells than did the non-SP cells, $38.7 \% \pm 2.5 \%$ and $9.5 \% \pm 1.1 \%$, respectively, and a higher proportion of PE-CAM-1-expressing cells, $19.8 \% \pm 8.0 \%$ and $4.6 \% \pm 3.5 \%$, respectively. Flk- 1 and VE-cadherin were not detectable in either population; CD34 was only detectable in the non-SP population and expressed at low levels $(10-15 \%)$. Cytospin analysis revealed expression of PDGFR- $\beta$ and SM- $\alpha$-actin, but not calponin or desmin, proteins in non-SP cells; SP cells expressed none of these proteins. RNA and protein expression data for muscle SP and non-SP cells, as well as previously published expression data from bone marrow SP cells $(4,12)$, are summarized in Table 1.

Vascular progenitors resident in skeletal muscle are derived from bone marrow. Since SP cells from bone marrow and skeletal muscle were phenotypically distinct, we aimed to determine whether muscle SP cells were derived from bone marrow and address whether tissue environment influences their vascular potential. Thus, we transplanted lethally irradiated recipients with Rosa26derived bone marrow SP cells. After 5 or 12 months, skeletal muscle tissue was excised, digested, and subjected to a $40-70 \%$ Percoll gradient. Percoll-fractionated muscle cells were then stained with Hoechst dye and loaded with FDG, to isolate muscle SP and non-SP cells from the stably engrafted mice and detect $\beta$-gal activity (LacZ expression) within each population, respectively. Both muscle SP and non-SP populations were found to be largely derived from bone marrow SP cells, as evidenced by their $\beta$-gal activity (Figure 4 ). Furthermore, the proportion of marrow-derived cells within these skeletal muscle populations significantly increased over time. At 5 months after transplantation (Figure 4a), the average number of SP and non-SP cells derived from LacZ-positive bone marrow SP cells, among three mice, was $39.0 \% \pm 16.4 \%$ and $10.6 \% \pm 1.9 \%$, respectively. At 12 months after transplantation (Figure $4 \mathrm{~b}$ ), $89.4 \% \pm 8.2 \%$ SP cells and $87.5 \% \pm 5.2 \%$ non-SP cells were LacZ positive and, therefore, derived from engrafted LacZ-positive bone marrow SP cells. Of the approximately $90 \%$ LacZ-positive non-SP cells found within muscle tissue 12 months after transplantation, $22.5 \% \pm 3.2 \%$ were CD45 negative.

Muscle-derived progenitors differentially contribute to the regeneration of vascular endothelium and smooth muscle. To test the vascular potential of skeletal muscle-derived SP and non-SP cells, genetically marked (LacZ-positive) cells were injected into skeletal muscle undergoing injury-induced neovascularization. Four weeks after injury of the TA muscle, the lower hindlimb musculature was harvested and stem cell engraftment examined using immunohistochemical techniques. Histological staining revealed muscle injury, as evidenced by misalignment of muscle fibers and excessive spacing between fibers, throughout the entire length of the TA muscle, as expected given that the cardiotoxin was injected lengthwise; approximately $50 \%$ of the tissue area on cross-section exhibited evidence of injury. The precise border of the injured region could not always be

\section{Table 1}

Summary of FACS and RT-PCR analyses

\begin{tabular}{lccc}
\hline & BM SP & M SP & M non-SP \\
Multipotent progenitor & Tal-1 & - & - \\
& VEGF-A & - & - \\
& - & c-met & c-met \\
& CD45 & CD45 & CD45 \\
& Sca-1 & Sca-1 & Sca-1 \\
& PE-CAM & PE-CAM & PE-CAMA \\
& Tie-2 & Tie-2 & Tie-2A \\
EC lineage & - & - & CD34 \\
SM lineage & - & - & - \\
& - & - & SM- $\alpha$-actin \\
& & & Calponin \\
& & & PDGFR $\beta$
\end{tabular}

Results from all RT-PCR and FACS analyses, as well as immunostaining of cytospin preparations, are summarized and compared with previously published bone marrow SP cell expression of these genes and proteins. The level of expression in bone marrow SP cells was previously determined to be: CD 45 , approximately $95 \%$, and Sca-1, approximately $98 \%$ (12); PE-CAM, approximately $98 \%$, and Tie- 2 , approximately $70 \%$ (4). AFewer than $10 \%$ of the cells in the population expressed the protein, as determined by FACS. ${ }^{B} \mathrm{M}$ non-SP expressed only mRNA for calponin, but not protein. BM, bone marrow; $M$, muscle. 
a 5 months after transplant

$\mathrm{SP}$

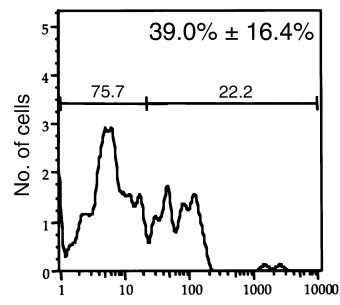

b 12 months after transplant $\mathrm{SP}$

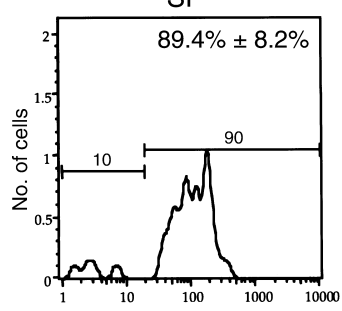

FDG

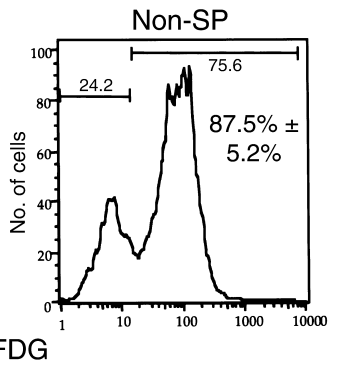

clearly delineated; therefore, quantification of stem cell engraftment was based on total number of vessels throughout the entire TA muscle. Thus, the engraftment levels reported herein may under represent stem cell contributions to neovascularization in the most extensively injured muscle tissue.

We found that muscle-derived SP cells had incorporated into the endothelium of small caliber vessels (Figure 5, a and b), as revealed by coexpression of $\beta$-gal (Figure 5, $\mathrm{c}$ and $\mathrm{d}$ ) and endothelial marker ICAM-2 (Figure 5, e and $\mathrm{f}$ ). The merger of $\beta$-gal and ICAM-2 staining patterns reveals colocalization (yellow color) in vessel structures (Figure 5, g and h). The number of ICAM-2-positive vessel structures that were also positive for $\beta$-gal in each of four animals was: 25 out of $1,014,39$ out of 768,31 out of 646 , and 18 out of 424 vessel structures, respectively. Thus, the mean proportion of vessels in the TA muscle that exhibited SP-

\section{Figure 5}

Skeletal muscle-derived SP cells engraft into vascular endothelium. Six- to eight-week-old C57BI/ 6 mice were anesthetized with Avertin, and both TA muscles were injected with $25 \mu \mathrm{l}$ of a $1 \mathrm{mg} / \mathrm{ml}$ cardiotoxin. After 8 or 24 hours, the right limb TA muscle was injected with 2,000 LacZ-positive muscle SP cells, and the left leg was injected with HBSS alone. After 4 weeks, the TA muscles of each of four experimental animals were harvested and processed for frozen sectioning and immunohistochemical analysis. All sections were immunostained for $\beta$-gal and costained for either ICAM- 2 or desmin to detect injury-induced engraftment of LacZ-positive SP cells into regenerated vascular endothelium and smooth muscle, respectively. $\beta$-gal expression in vascular structures was colocalized only with ICAM-2: ( $\mathbf{a}$ and $\mathbf{b}$ ) bright field, boxes indicate costained vessels; (c and d) $\beta$-gal immunohistochemistry; (e and f) ICAM- 2 immunohistochemistry; ( $\mathbf{g}$ and $\mathbf{h}$ ) $\beta$-gal and ICAM- 2 fluorescent images were merged to reveal areas of costaining, as evidenced by the yellow staining pattern. Magnification, $\times 400(\mathbf{a}, \mathbf{c}, \mathbf{e}, \mathbf{g}) ; \times 1,000(\mathbf{b}, \mathbf{d}, \mathbf{f}, \mathbf{h}$, and inset boxes in $\mathbf{c}, \mathbf{e}$, and $\mathbf{g}$ ).

\section{Figure 4}

Vascular progenitors within skeletal muscle are derived from bone marrow. SP cells were isolated from bone marrow of Rosa 26 mice and stably engrafted into lethally irradiated, genetically matched recipients. After 5 or 12 months, the hindlimb musculature was excised and examined for the presence of LacZ-positive vascular progenitors. Percoll-fractionated muscle cells were stained with Hoechst dye and loaded with FDG, then subjected to FACS to isolate muscle SP and non-SP populations and determine whether they exhibit $\beta$-gal activity. (a) The mean \pm SD of LacZ-positive SP and non-SP cells among three mice at 5 months after transplant was $39.0 \% \pm 16.4 \%$ and $10.6 \% \pm 1.9 \%$, respectively. (b) At 12 months after transplantation, the mean \pm SD of LacZ-positive SP and non-SP cells among three mice was $89.4 \% \pm 8.2 \%$ and $87.5 \% \pm 5.2 \%$, respectively.

derived endothelial cells was $4.2 \% \pm 1.0 \%$. In these same animals, engraftment of marked stem cells was not detected in vessel structures in noninjured hindlimb muscles. Furthermore, no $\beta$-gal-positive cells were colocalized with the smooth muscle marker desmin $(0 / 2,300$ vessel structures examined among four animals) in any region of the musculature; thus, SP cells did not engraft into the smooth muscle layer of vessels in these tissues.

Conversely, $\beta$-gal-positive, muscle-derived non-SP cells exhibited engraftment into the smooth muscle layer of large- (Figure 6, a-f) and small-caliber vessels (Figure 6, $\mathrm{g}-\mathrm{j}$ ), and costained with the smooth muscle marker
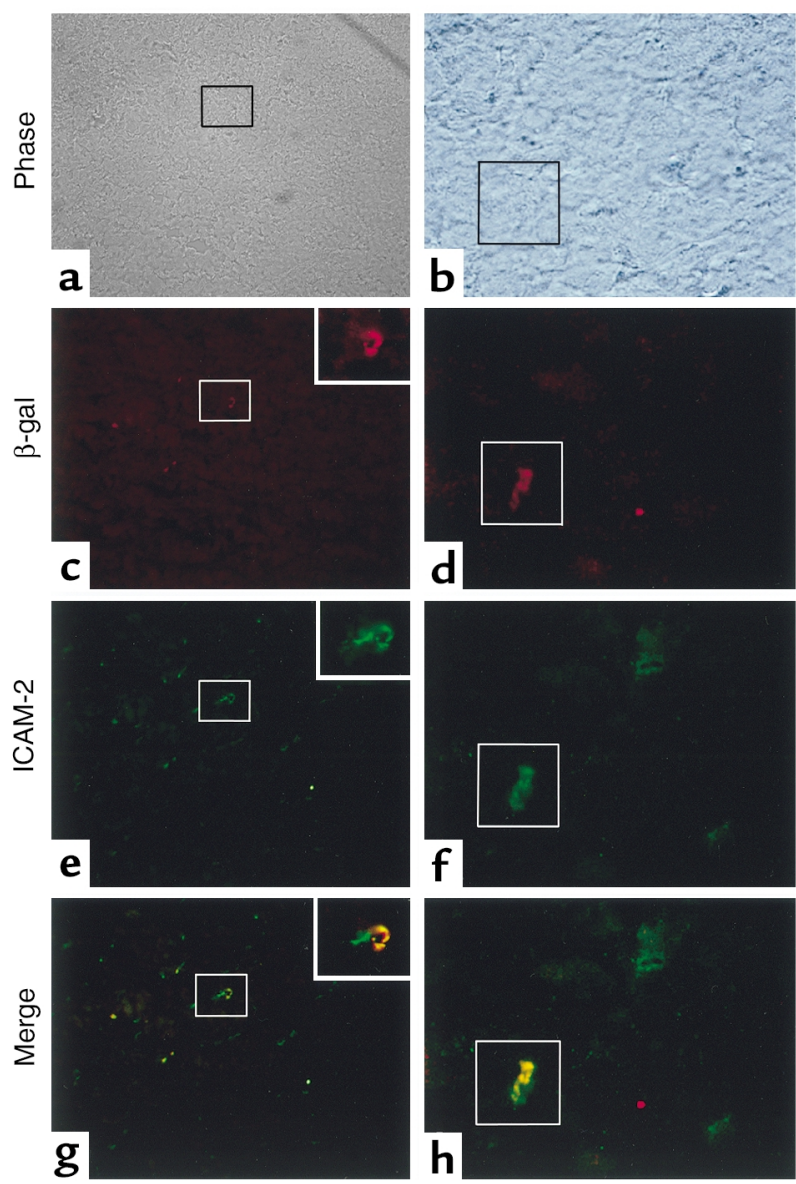

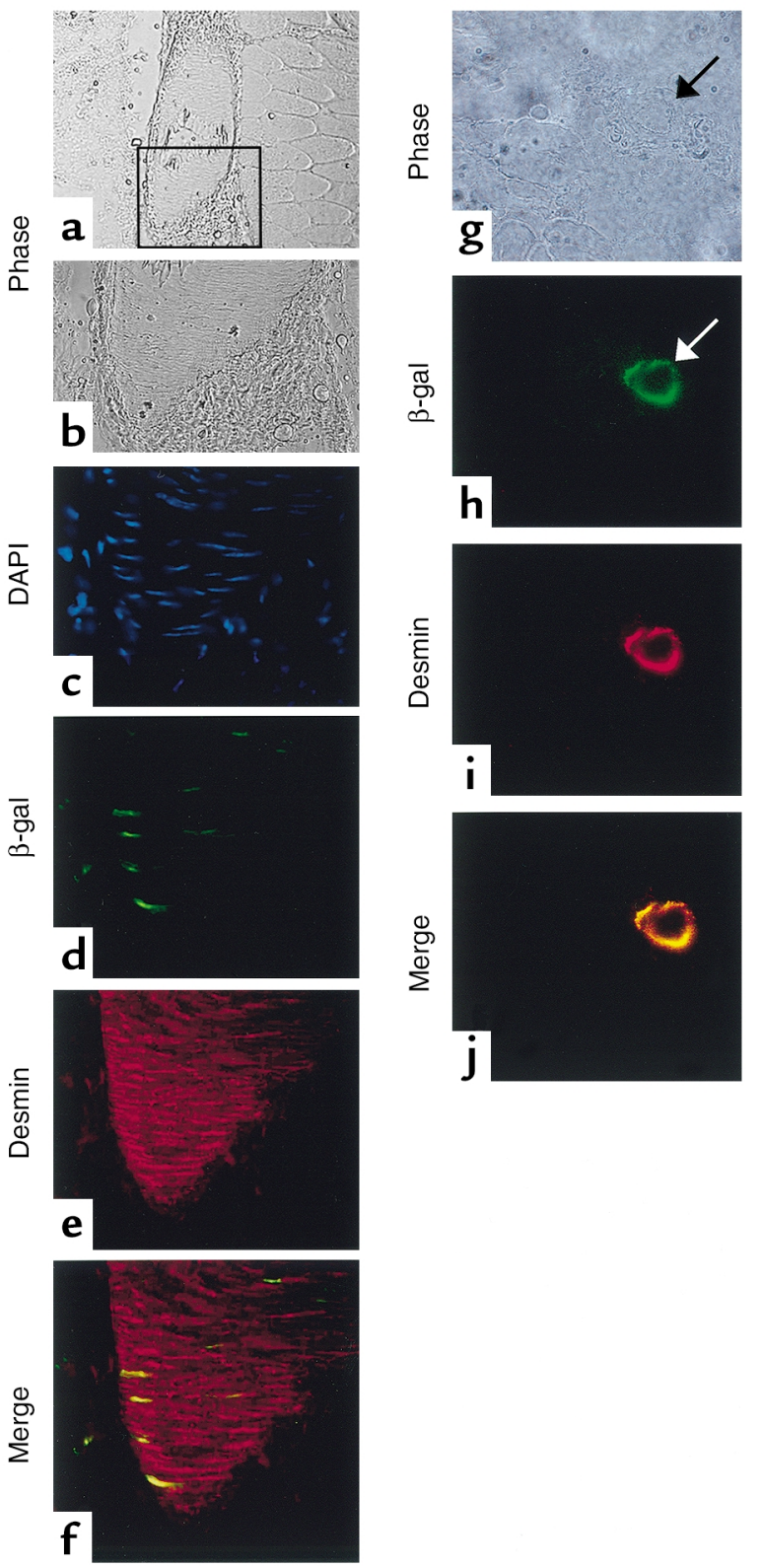

desmin (Figure 6, e and i). The merger of $\beta$-gal and desmin staining patterns reveals colocalization (yellow color) in vessel structures (Figure 6, f and j). In general, the total number of smooth muscle cells, identified by desmin-positive staining, was fewer than the number of identifiable endothelial cells in these muscle tissue sections. Therefore, the number of countable vessel structures is concomitantly lower in these experiments, relative to those described above. Among four experimental animals, the number of desmin-positive vessel structures that were also positive for $\beta$-gal was 59 out of 567 , or $10.3 \% \pm 4.4 \%$ engraftment. No $\beta$-gal-positive non-SP cells were found in the ICAM-2-positive endothelial layer of vessel structures (0/875); thus, non-SP cells did not engraft into the vascular endothelium. We found no $\beta$-gal-positive cells in the skeletal muscle or vasculature $(0 / 2,000$ vessel structures) of sham-treated animals.

\section{Figure 6}

Skeletal muscle-derived non-SP cells engraft into vascular smooth muscle. Six- to eight- week-old $\mathrm{C} 57 \mathrm{BI} / 6$ mice were anesthetized with Avertin, and both TA muscles were injected with $25 \mu \mathrm{l}$ of a $1 \mathrm{mg} / \mathrm{ml}$ cardiotoxin. After 8 or 24 hours, the right limb TA muscle was injected with 500,000 LacZ-positive muscle non-SP cells, and the left leg was injected with HBSS alone. After 4 weeks, the TA muscles of each of four experimental animals were harvested and processed for frozen sectioning and immunohistochemical analysis. All sections were immunostained for $\beta$-gal, and costained for either ICAM- 2 or desmin to detect injury-induced engraftment of LacZ positive non-SP cells into regenerated vascular endothelium and smooth muscle, respectively. $\beta$-gal expression in vascular structures colocalized only with desmin: (a, b, and $\mathbf{g}$ ) bright field, box in $\mathbf{a}$ indicates region shown in (b-f); (c) DAPI; (d and h) $\beta$-gal immunohistochemistry; (e and i) desmin immunohistochemistry; ( $f$ and $\mathbf{j}$ ) $\beta$-gal and desmin fluorescent images were merged to reveal areas of costaining, as evidenced by the yellow staining pattern. Magnification, $\times 200(\mathbf{a}) ; \times 600$ (b, c, $\mathbf{d}, \mathbf{e}$, and $\mathbf{f}) ; \times 1,000(\mathbf{g}, \mathbf{h}, \mathbf{i}$, and $\mathbf{j})$.

\section{Discussion}

In these studies, we aimed to determine whether vascular progenitors reside within adult skeletal muscle, whether they are derived from bone marrow progenitors, and to what extent they contribute to injuryinduced vascular regeneration in muscle.

From skeletal muscle, we isolated a highly purified population of hematopoietic stem cells, SP cells, as well as a non-SP cell population, which does not reconstitute blood and contains mesenchymal progenitors (15). We determined that the majority of both of these distinct populations resident within skeletal muscle were replenished over time from bone marrow stem cells (bone marrow SP cells). Furthermore, our data suggest that the muscle-derived SP and non-SP cells exhibit different cell fates during injury-induced regeneration, forming vascular endothelium and smooth muscle, respectively.

These findings are consistent with our previous bone marrow studies (4), indicating that stem cell populations with hematopoietic activity (SP cells) also can generate vascular endothelium. From the present studies, it is clear that there is a second distinct and reproducibly isolated progenitor population (non-SP cells) resident within skeletal muscle that is mesenchymal rather than hematopoietic in nature and contributes to the regeneration of vascular smooth muscle, but not endothelial, cells during injury-induced neovascularization. Given the mesenchymal characteristics of the non-SP population, it was not surprising that non-SP cells contributed only to the regeneration of vascular smooth muscle cells and not endothelium. That is, the differentiation of endothelial cells from mesenchymal lineages has not been observed previously, although the generation of mesenchymal lineages from endothelium has been reported $(20,21)$.

Consistent with this idea, our own previous studies demonstrate that bone marrow SP cells that regenerate endothelial cells, also regenerate cardiac muscle cells, albeit at a very low frequency $(\sim 0.02 \%)$ (4). Thus, the fact that we did not observe smooth muscle cell regen- 
eration from muscle SP cells was somewhat unexpected. It is possible that muscle SP engraftment into vascular smooth muscle would be observed after longer periods of postinjury repair. Since our data suggest that SP cells within bone marrow regenerate the nonSP population within muscle that contains mesenchymal progenitors, it is possible that SP-derived non-SP cells would serve as smooth muscle progenitors during injury-induced neovascularization in muscle.

It is interesting that both populations of distinct vascular (endothelial and smooth muscle) progenitors that we identified within skeletal muscle were found to be increasingly LacZ positive over time, suggesting that both were largely derived from and replenished by engrafted LacZ-marked bone marrow SP cells. Thus, it is tempting to speculate that both vascular endothelial and smooth muscle progenitors within nonmarrow tissues are ultimately derived from a common progenitor within bone marrow. Alternatively, the two different populations could be derived from distinct progenitors within the bone marrow SP fraction. However, considering that bone marrow SP cells are $95-98 \%$ similar in phenotype, as evidenced by flow cytometric analysis for cell surface markers (ref. 12; and Figure 3, legend), this latter scenario would require that one vascular progenitor population (presumably the nonhematopoietic non-SP population) be derived from a distinct progenitor contained within $2-5 \%$ of the engrafted bone marrow SP. This issue remains to be directly addressed via single cell transplantation studies.

The level of engraftment of muscle-derived vascular progenitors into newly forming and/or remodeling vasculature reported herein is consistent with our previous studies demonstrating that $3-5 \%$ of endothelial cells regenerated in response to injury are derived from bone marrow stem cells (4). The level of engraftment of non-SP cells into muscle was higher $(-10 \%)$ and consistent with reports from other labs (3). Interestingly, other studies report levels of bone marrow stem cell engraftment into vascular cells to be as high as $40-50 \%$ in response to injury (5). These apparent differences in observed contributions of stem cells to injury-induced neovascularization may reflect differences in quantification techniques, differences in the contribution of unmarked endogenous stem cells within the injured tissue, and/or differences in the vascular potential of the specific stem cell populations studied.

In our recent studies (ref. 4; this study), the absolute contribution of stem cells to vascular regeneration may be underrepresented, since the quantification of stem cell engraftment was based on total number of vessels throughout the entire muscle (cardiac and TA, respectively), even though some areas were not acutely injured. Others quantify engraftment based on number of labeled cell nuclei versus total cell nuclei in a specific region of injury (5). However, even in this system, where injured tissue may be more clearly defined, the contribution of endogenous (unmarked) stem cells is still not measured or limited. Thus, no quantification technique defined to date can determine precisely the contribution of stem cells to injury-induced neovascularization.

Regardless of absolute engraftment levels, our data demonstrate that distinct populations of vascular endothelial and smooth muscle progenitors could be purified from skeletal muscle, indicating that nonmarrow adult tissue could prove to be a clinically important source of cells for autologous vascular cell therapies and tissue engineering. Moreover, since tissue-resident vascular progenitors can be regenerated from transplanted bone marrow stem cells, transplantation of allogeneic or autologous genetically altered bone marrow may prove to be a useful treatment strategy or adjunct therapy for common vascular pathologies resulting from defective regenerative capacity, including coronary and peripheral ischemia.

Optimization of such treatment strategies would require a better understanding of the deposition, survival, and release of such vascular progenitors from their tissue residence, as well as a more thorough characterization of the molecular profile of selected target populations. Toward that end, we directly compared the expression of genes thought to play a role in blood vessel formation in SP cells isolated from bone marrow and muscle. Interestingly, although the SP cells within skeletal muscle and bone marrow share a common origin and exhibit similar capacity to regenerate endothelium, they exhibited phenotypic differences within their tissue environment, which provides support for the theory that microenvironment (or "niche") dictates stem cell and progenitor phenotype (22), and perhaps behavior.

The most notable distinction between marrow- and muscle-derived SP cells is that muscle SP cells lacked the expression of Tal1 and VEGF-A, although both SP populations expressed $P E-C A M-1$ and Tie-2, consistent with embryonic endothelial (23) and blood (17) progenitors. The lack of detectable Tal1 gene expression in muscle SP cells is surprising, because its expression was found to be essential for embryonic hematopoiesis (24), yet muscle SP cells reconstitute blood in adults (ref. 11, S. McKinney-Freeman et al., manuscript submitted for publication). Tal1 is also expressed by embryonic progenitors destined to become endothelial cells (25), although its expression was not found to be necessary for endothelial cell differentiation (24).

VEGF-A is expressed by bone marrow SP cells but not by muscle SP cells. Since muscle- and marrow-derived SP cells regenerate endothelium to a similar extent, it appears that the expression of VEGF-A has no effect on the endothelial potential of SP cells. However, it is possible that VEGF-A expression in bone marrow SP cells enables survival within the marrow environment, as has been recently shown for other hematopoietic stem cell populations (26), as well as other cell types (27).

Regardless of phenotypic differences between muscle- and marrow-derived SP cells, it is clear that the phenotype of the muscle SP population, as a whole, is distinctly different from that of the muscle non-SP population. Specifically, muscle SP cells share charac- 
teristics with hematopoietic and multipotent stem cells, as well as embryonic vascular progenitors, as discussed above, whereas the non-SP cells exhibit characteristics of mesenchymal cells (15) and mesenchymal stem cells (28) that are not exhibited by either the bone marrow or muscle SP cells. Based on its phenotype, it is possible that the murine muscle non-SP population contains bona fide mesenchymal stem cells that are capable of regenerating multiple mesenchymal lineages (bone, adipose, cartilage) in the appropriate microenvironment(s), as has been described for human skeletal muscle (29), but this remains to be determined. In summary, although it is unclear at present how the above differences in gene expression between distinct vascular progenitor populations within skeletal muscle affects their survival and long-term function, it is clear that some phenotypic characteristics can aid in predicting a progenitor's fate toward a vascular lineage. That is, a SM- $\alpha$-actin-expressing progenitor is not likely to generate an endothelial cell. Such information will be very useful for choosing target progenitor populations, as well as defining needed genetic alterations to direct fate. Our progress in identifying and characterizing the potential of vascular progenitors within adult skeletal muscle and bone marrow provides insights needed for achieving these goals.

\section{Acknowledgments}

We thank Mike Cubbage and Brian Newsom for expert assistance with FACS. We thank Shannon McKinneyFreeman, Brian Nadin, and Chelsea Wilson for their technical assistance, and all members of our lab for helpful discussions. S.M. Majka is supported by NIH grant NRSA F32-HL68482. K.A. Jackson is a fellow of the Leukemia and Lymphoma Society. M.W. Majesky is an Established Investigator of the American Heart Association (AHA-150217). M.A. Goodell is a Leukemia and Lymphoma Society Scholar. Grants to K.K. Hirschi that supported this work include NIH R01-HL61408, USDA 6250-51000-033, AHA-National SDG 9930054N, and the Gillson Longenbough Foundation Award.

1. Asahara, T., et al. 1997. Isolation of putative progenitor endothelial cells for angiogenesis. Science. 275:964-967.

2. Schatteman, G.C., et al. 2000. Blood-derived angioblasts accelerate blood flow restoration in diabetic mice. J. Clin. Invest. 106:571-578.

3. Shimizu, K., et al. 2001. Host bone marrow cells are a source of donor intimal smooth muscle-like cells in murine aortic transplant arteriopathy. Nat. Med. 7:738-741.

4. Jackson, K.A., et al. 2001. Regeneration of cardiac muscle and vascular endothelium by adult stem cells. J. Clin. Invest. 107:1395-1402.
5. Orlic, D., et al. 2001. Bone marrow cells regenerate infarcted myocardium. Nature. 410:701-705.

6. Orlic, D., et al. 2001. Mobilized bone marrow cells repair the infarcted heart, improving function and survival. Proc. Natl. Acad. Sci. USA. 98:10344-10349.

7. Sata, M., et al. 2002. Hematopoietic stem cells differentiate into vascular cells that participate in the pathogenesis of atherosclerosis. Nat. Med. 8:403-409.

8. Niklason, L.E., et al. 1999. Functional arteries grown in vitro. Science. 284:489-493.

9. Morgan, J.E., Coulton, G.R., and Partridge, T.A. 1987. Muscle precursor cells invade and repopulate freeze-killed muscles. J. Muscle Res. Cell Motil. 8:386-396.

10. Jackson, K.A., Mi, T., and Goodell, M.A. 1999. Muscle stem cells can function as hematopoietic stem cells. Proc. Natl. Acad. Sci. USA. 96:14482-14486

11. Gussoni, F., et al. 1999. Dystrophin expression in the mdx mouse restored by stem cells transplantation. Nature. 401:390-404.

12. Goodell, M.A., Brose, K., Paradis, G., Conner, A., and Mulligan, R. 1996. Isolation and functional properties of murine hematopoietic stem cells that are replicating in vivo. J. Exp. Med. 183:1797-1806.

13. Sato, T.N., Qin, Y., Kozak, C., and Audus, K.L. 1993. tie-1 and tie-2 define another class of putative receptor tyrosine kinase genes expressed in early embryonic vascular system. Proc. Natl. Acad. Sci. USA. 90:9355-9358.

14. Couteaux, R., Mira, J.C., and d'Albis, A. 1988. Regeneration of muscles after cardiotoxin injury. I. Cytological aspects. Biol. Cell. 62:171-182.

15. Hirschi, K.K., Rohovsky, S.A., and D'Amore, P.A. 1998. PDGF, TGF- $\beta$ and heterotypic cell-cell interactions mediate the recruitment and differentiation of $10 \mathrm{~T} 1 / 2$ cells to a smooth muscle cell fate. J. Cell Biol. 141:805-814

16. White, T.P., and Esser, K.A. 1989. Satellite cell and growth factor involvement in skeletal muscle growth. Med. Sci. Sports Exerc. 21:5158-5163.

17. Keller, G. 2001. The hemangioblast. In Stem cell biology. D.R. Marshak, R.L. Gardner, and D. Gottlieb, editors. Cold Spring Harbor Laboratory Press. Cold Spring Harbor, New York, USA. 329-341.

18. Drake, C.J., and Fleming, P.A. 2000. Vasculogenesis in the day 6.5 to 9.5 mouse embryo. Blood. 95:1671-1679.

19. Hirschi, K.K., and Goodell, M.A. 2001. Common origins of blood and blood vessels in adults? Differentiation. 68:186-192.

20. DeRuiter, M.C., et al. 1997. Embryonic endothelial cells transdifferentiate into mesenchymal cells expressing smooth muscle actin in vivo and in vitro. Circ. Res. 80:444-451.

21. Condorelli, G., et al. 2001. Cardiomyocytes induce endothelial cells to transdifferentiate into cardiac muscle: Implications for myocardium regeneration. Proc. Natl. Acad. Sci. USA. 96:10733-10738.

22. Spradling, A., Drummond-Barbosa, D., and Kai, T. 2001. Stem cells find their niche. Nature. 414:98-104.

23. Hatzopoulos, A.K., Folkman, J., Vasile, E., Eiselen, G.K., and Rosenberg, R.D. 1998. Isolation and characterization of endothelial progenitor cells from mouse embryos. Development. 125:1457-1468.

24. Robb, L., et al. 1995. Absence of yolk sac hematopoiesis from mice with a targeted disruption of the $s c l$ gene. Proc. Natl. Acad. Sci. USA. 92:7075-7079.

25. Drake, C.J., Brandt, S.J., Trusk, T.C., and Little, C.D. 1997. Tal1/SCL is expressed in endothelial progenitor cells/angioblasts and defines a dorsal-to-ventral gradient of vasculogenesis. Dev. Biol. 192:17-30.

26. Gerber, H.-P., et al. 2002. VEGF regulates hematopoietic stem cell survival by an internal autocrine loop mechanism. Nature. 417:954-958.

27. Alon, T., Hemo, I., and Itin, A. 1995. Vascular endothelial growth factor acts as a survival factor for newly formed retinal vessels and has implications for retinopathy of prematurity. Nat. Med. 1:1024-1028.

28. Arakawa, E., Hasegawa, K., Yanai, N., Obinata, M., and Matsuda, Y. 2000. A mouse bone marrow stromal cell line, TBR-B, shows inducible expression of smooth muscle-specific genes. FEBS Lett. 481:193-196.

29. Young, H.E., et al. 2001. Human reserve pluripotent mesenchymal stem cells are present in the connective tissues of skeletal muscle and dermis derived from fetal, adult, and geriatric donors. Anat. Rec. 264:51-62. 